\title{
Wearable and Augmented Reality Displays Using MEMS and SLMs
}

\author{
Hakan Urey*, Erdem Ulusoy, Seyedmahdi M. K. Kazempourradi, Deniz Mengu, \\ Selim Olcer, Sven T. Holmstrom \\ Optical Microsystems Laboratory, Koç University, Department of Electrical and \\ Electronics Engineering, TR-34450, Sarıer, İstanbul, Turkey
}

\begin{abstract}
In this talk, we present the various types of 3D displays, head-mounted projection displays and wearable displays developed in our group using MEMS scanners, compact RGB laser light sources, and spatial light modulators.
\end{abstract}

Keywords: 3D Displays, Wearable Displays, MEMS, Pico Projector, Spatial Light Modulator

\section{INTRODUCTION}

Human-computer interaction involves exchange of information between a human and a computer. It is relatively easy to convey the messages from the human to the computer side, since the 'receptors' of a computer are man-made, and it is possible to devise and continuously improve lots of mechanisms such as keyboards, mouse, microphones, touch-screens, gamepads, etc. depending on emerging needs and applications. The options for the reverse direction, however, are limited: humans have only five senses, and a computer must eventually address one of these. Since sight is the primary sense through which humans gather information about the external world, as emphasized in the famous anonymous quotation 'one picture is worth more than ten thousand words', displays constitute the most integral component of human-computer interaction, and it seems that they will continue to do so till solutions that directly stimulate the visual cortex of the brain make them obsolete.

Beginning from 1950s, electronic displays became commonplace with the cathode ray tube (CRT) technology. Due to the initial high cost of the technology, CRTs were necessarily designed to provide large screen size and large viewing angle so that multiple users can be served at a time. In addition, the bulky back tubes of CRTs necessitated fixed installations. As the technology became cheaper around 1980s, personal displays such as desktop monitors became affordable. The bulkiness of the display, though, still restricted its usage to immobile indoor applications. Beginning with the new millennium, the rise of the flat-panel LCD, plasma or LED displays has eliminated the bulk of back tubes, and initiated a new trend towards enhanced mobility, along with the advancement in miniaturization of electronic circuitry. Indeed, it took a minor time for smaller size high quality flat-panel displays to be developed and integrated with now vastly enjoyed mobile devices such as smart phones, laptop and tablet computers.

Interestingly, throughout all the mentioned period, conceptualization of displays essentially remained the same, i.e. display functions as the screen on which the images are shown, display has a large viewing angle, and serves multiple users at a time. Today's conditions and expectations, however, force us to redeem several aspects of this paradigm. One concern is about the tradeoff that has emerged between productivity and mobility. In particular, people work less productively on the small displays of mobile devices that provide limited field of view (FOV). For instance, while browsing the internet on a smart phone, we usually need to frequently perform zoom in-outs. Mobile devices also disturb hand freedom and level of attention, for instance, while driving. In comparison, larger size displays are much easier to work with. However, large displays, even if flat-panel, are bulky and impede mobility. Conventional projectors resolve this compromise to some degree, yet they are still hardly suitable for personal use in mobile and outdoor applications.

A second concern is related to light efficiency. For most of its lifetime the display of a personal mobile device is viewed only by a single user. In typical mobile devices, the display consumes almost half of the battery power, of which merely about $0.1 \%$ of the display light enters through the eye pupils of a user at a time. The remaining light, thus the associated portion of the battery power, is simply wasted if no other viewers are present. To further elucidate this inefficiency, note that images on smart phones are poorly visible under bright sunlight. Yet, if the total power emanated by the display (around 2-3 Watts) would be collected and directed into the eye, the irradiance levels will be well above safety limits.

MOEMS and Miniaturized Systems XV, edited by Wibool Piyawattanametha,

Yong-Hwa Park, Proc. of SPIE Vol. 9760, 976004 - (c) 2016 SPIE

CCC code: $0277-786 \mathrm{X} / 16 / \$ 18 \cdot$ doi: $10.1117 / 12.2216633$ 
Such great light inefficiencies definitely require a reconsideration of existing display paradigms, especially when we take into account the abundance of low-cost, high performance, and miniaturized sensor technologies.

For over a decade, our laboratory has been engaged with the development of various new optical components, systems, and algorithms with an objective of devising display solutions that simultaneously provide productivity, mobility and light efficiency. In the recent years, following the emerging trends, we have also incorporated wearable augmented reality and three dimensional display schemes in our agenda. In this paper, we provide a summary of our progress reported elsewhere along with some of the latest results.

\section{MEMS LASER SCANNER BASED SOLUTIONS}

Pico-projectors provide a good solution towards productive, mobile and light efficient displays. Our lab has been at the forefront of research in MEMS scanner development. First pico-projector product ShowWX using MEMS scanners was launched by Microvision Inc in 2009 as illustrated in Fig. 1a [1]. This product establishes a connection with a smart phone, and projects the display content on a larger screen. With this solution, users are no longer restricted by a small display and can work much more productively with the projected large size image. The inclusion of the small projector hinders mobility only minimally. Other noteworthy features of the miniature projector are its focus free operation due to the emission of highly directive laser light, and very rich color gamut. 3D PicoP is also possible using an active polarization rotator and passive glasses using the mixed polarization 3D technique we developed [2].

To further enhance productivity, we considered an arrangement in the form of a head mounted projection display (HMPD) as shown in Fig.1b [3]. Such an arrangement provides hand freedom that might be desirable in applications such as surgery, driving and gaming. The system however, still suffers from light inefficiency, especially if projections are made on diffuse surfaces. Usage of special screens, if the application and the settings permit, can significantly improve light efficiency. An option is to use retroreflector screens, which essentially reflect incident light mainly towards the source. When the pico-projector is placed close to the eye, virtually all the light emitted by the projector is directed to the eye, and no light is lost to surroundings. Retroreflector screens are already available in the form of easily foldable and portable lightweight fabrics. Since a viewer's projection on a retroreflective screen is only visible to her, common pre-installed screens can be shared among different users with negligible crosstalk. The increased light efficiency also enables sufficiently bright images visible under sunlight. Since retroreflector screens also maintain polarization, the HMPD can be extended to have 3D capability with minimum additional cost [2]. In particular, if a dynamic polarization rotator is deployed at the exit of the projector, left and right views can be polarization encoded, and then may simply be selected by a simple polarized glass, as in Fig.1c. In some settings such as airplanes, users sit fixed and essentially only have head motion freedom. Fig.1d illustrates a solution for such cases. Here, headset and glasses are removed, and two pico projectors are placed above the head. The retroreflective screen along with a vertical diffuser is mounted on a rotating platform. The combination creates two viewing slits (one per each eye) that can be steered to the position of viewers head. In this way, a light efficient autostereoscopic system is implemented [4].

Fig1.e shows a light efficient semi-transparent screen to be used with pico projectors [5]. Such screens are essential for emerging augmented reality applications such as automotive head up displays, where display image needs to be combined with real world view. The screen consists of a microlens array sandwiched between layers of UV curable epoxy. The pitch and tilt angles of the microlens array are adjustable to provide a desired eye box size. The screen is index matched so that real world view is not affected by the presence of microlenses. The visibility and transmissivity of the screen can be controlled by varying the level of metal coating on the lenses. The screen can be used from both sides simultaneously without crosstalk.

With display resolutions hitting full HD or even 4K, stereoscopic systems providing 3D visual experience has receiving a renewed interest [6]. In 3D display domain, several factors seem to impede further acceptance: visual discomfort associate with the so-called accommodation-convergence conflict, and the necessity to wear glasses. Expectations have also grown and now 3D displays providing more than two views, thus motion parallax are demanded. In a recent work, we proposed the usage of pinholes on the glasses, such that the depth of focus of the system is extended and accommodation response no longer creates a conflict with the convergence response [7]. When multiple pinholes are used per eye, a sense of blurring can also be provided for out of sight objects, hence a more natural 3D experience is delivered, though at the cost of worsened light efficiency. In another work, we implemented a multiview 3D system using an array of pico-projectors that can serve multiple glasses-free users simultaneously [8]. Here, pico-projectors are 
placed side by side, and a vertical diffuser is used to spread the light in vertical direction. Compared to a flat-panel display, the solution is much cheaper in hardware complexity, and also much better in resolution.

Eyewear displays integrated with smart tracking and gesture recognition sensors have the potential to completely resolve the compromise among mobility, productivity and light efficiency [9]. They can be easily portable while providing a large FOV. However, the fact that a human eye cannot focus directly on a near-to-eye display necessitates the usage of complicated relay lenses, imposing a compromise among FOV, resolution, image quality and form-factor. Eyewear displays with large FOV such as Oculus Rift or military headsets have limited resolution (in pixels/degree) or significant bulk, while those with attractive form-factor such as Google Glass have limited FOV. Fig.1f illustrates the product named NOMAD, launched in 2004 by Microvision Inc., where some of the underlying technologies were jointly developed with our laboratory. Fig.1g conceptually illustrates a more recent eyewear display project carried out in our laboratory. The display uses an unconventional scanning approach for image relay and to achieve augmented reality.

\section{a) Pico-projectors}

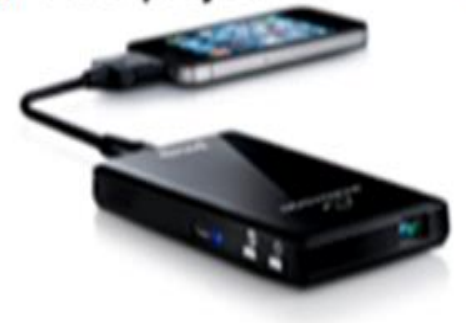

d)

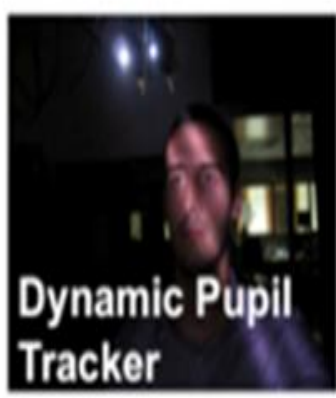

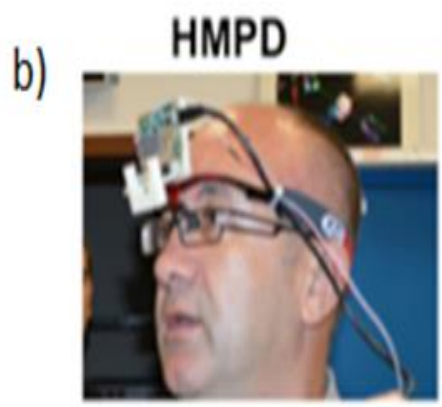

C)

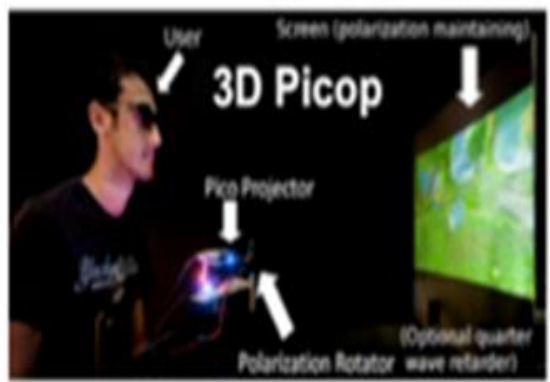

e)

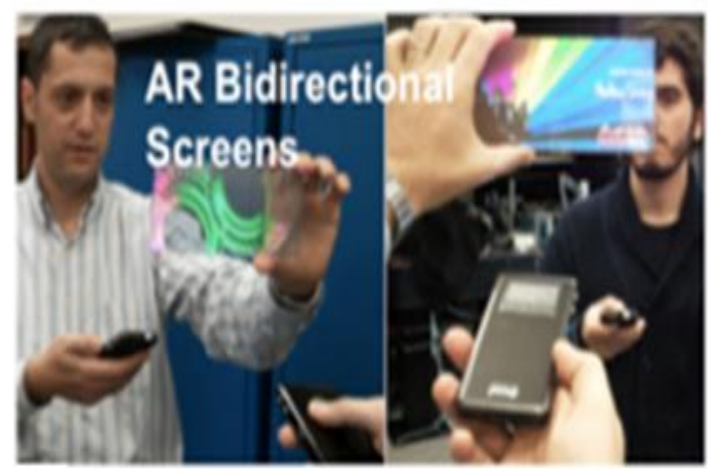

g)

\section{Eyewear displays}
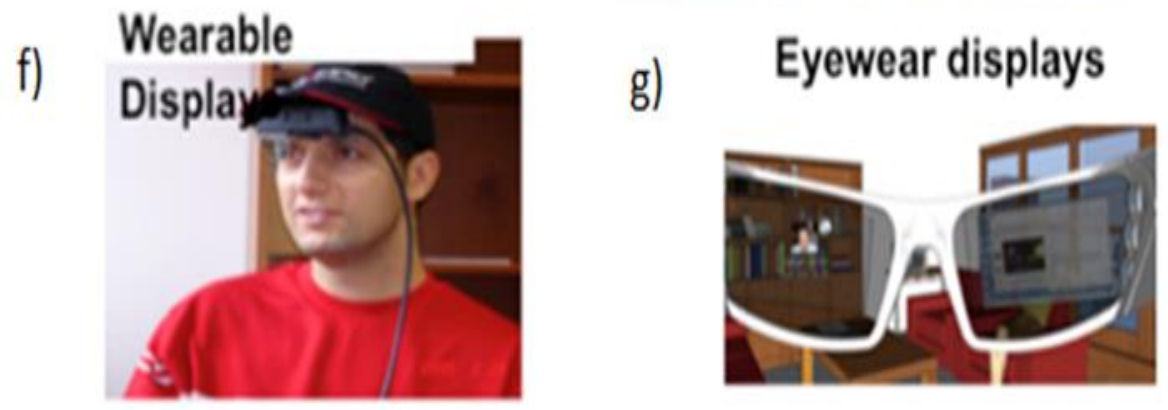

Figure 1. MEMS laser scanner based light efficient, mobile and productive display and screen technologies developed in Optical Microsystems Laboratory. 


\section{NOVEL FAST ALGORITHMS}

Another research track we pursue in our lab is to replace conventional projection systems with holographic projection systems employing spatial light modulators rather than microdisplays, since holographic systems offer unique capabilities and benefits over conventional systems such as soft aberration correction and increased light efficiency, which have the potential to enable more compact and robust holographic projectors for HUDs and other applications. However, holographic projection systems are disadvantageous in that they are prone to speckle noise, and computationally demanding [11]. In a recent work by our group, we developed an algorithm that dramatically reduces the computational complexity and improves the speckle performance of holographic projection systems that employ phase-only spatial light modulators [12].

Computational complexity of holographic projection systems is high because ideal holograms are in general complex valued while available light modulators perform a restricted type of modulation such as phase-only. The conversion of the full-complex hologram to a phase-only hologram while preserving image quality has customarily been performed by iterative algorithms such as iterative Fourier transform algorithm (IFTA), Gerschberg-Saxton, Direct Search, etc. Such algorithms mostly converge to a solution by allowing a phase freedom on image samples leading to speckle noise.

In our work, our first goal has been to free the computation from the burden of iterations. We started with the observation that two phase-only pixels together constitute a single degree of freedom. Then, we devised a method which utilizes simple Discrete Fourier Transform (DFT) properties to directly (in a non-iterative manner) compute a phase-only hologram that exactly reproduces half of the given image samples [13]. The remaining half of image samples is left uncontrolled and appears as encoding noise. The method merely requires a single Fast Fourier Transform (FFT) operation and several trivial block based operations. Following the basic method, we devised initial phase terms that are applied on the given image prior to the computation of the ideal hologram. The phase terms ensure that encoding noise appears outside the signal window on the image plane. Moreover, the non-random structure of the phase terms greatly reduced speckle noise. The mere drawback of the algorithm is that two SLM frames need to be temporally averaged to deliver progressive video, hence video frame rate equals half of the SLM frame rate. The system can readily deliver interlaced video format at the available SLM frame rate. Simulation results presented in Fig.4 verify the premises of our algorithm. Compared to commonly used iterative algorithms, our method provides a $20 \mathrm{X}$ times reduction in computational complexity. 
a)

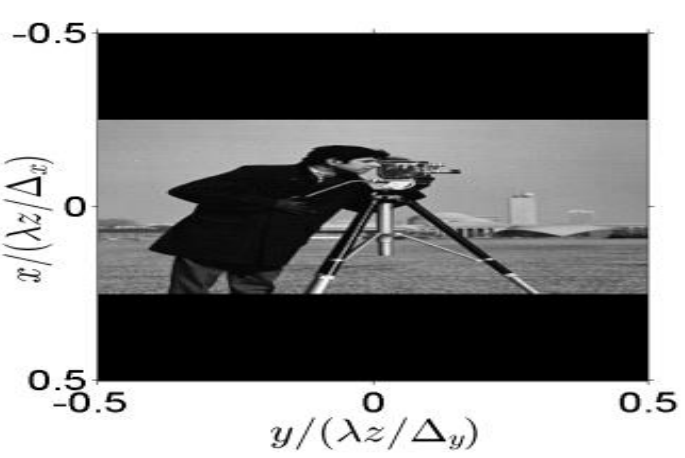

c)

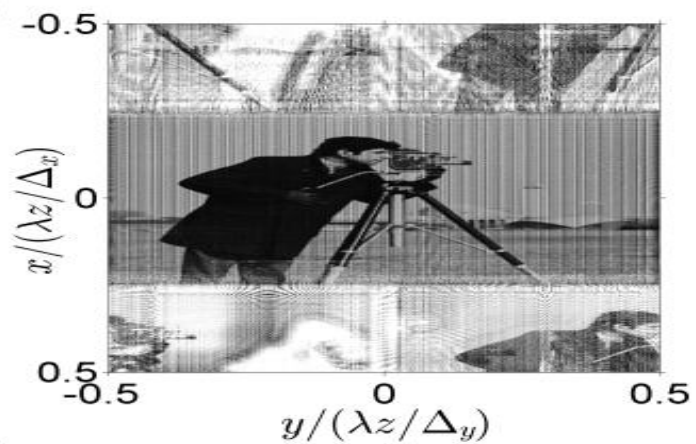

e)

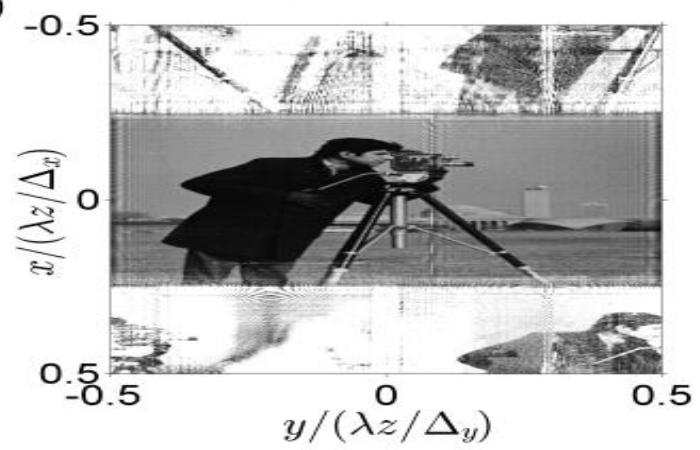

b)

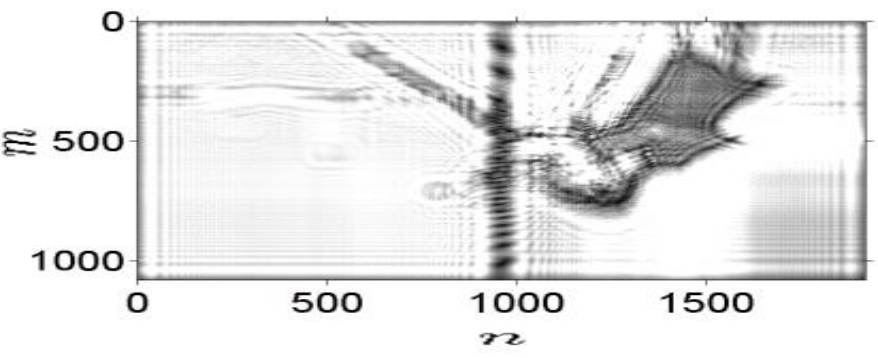

d)

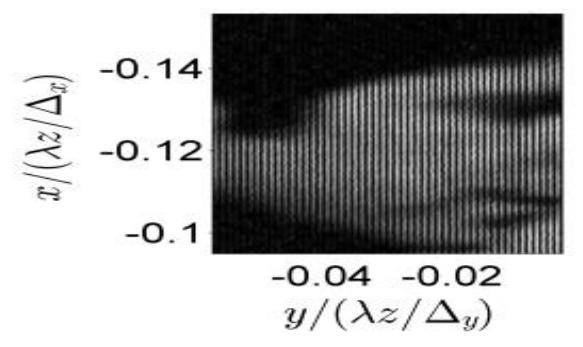

f)

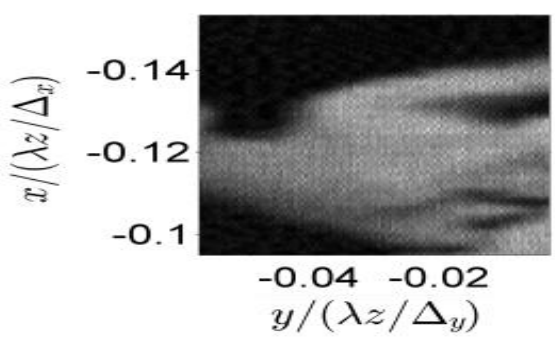

Figure 3. Fast non-iterative hologram computation method applied to holographic image projection. (a) Desired image. (b) Ideal full-complex hologram. (c) Reconstruction by a single phase hologram computed with the proposed method. Encoding noise is distributed to the region outside the image area. (d) Zoom-in version of c. Single hologram for each video frame supports interlaced video. (e) Temporal average of two SLM frames generates a complete video frame. (f) Zoom-in version of e. In the reconstructions speckle noise is quite low.

\section{ACKNOWLEDGEMENT}

This research was partly sponsored by Microvision Inc, Wear3D project funded by the European Research Council Advanced Grant (ERC-AdG) Project No: 340200 and TÜBİTAK Grant 111E183.

\section{REFERENCES}

[1] S. T. Holmstrom, U. Baran, H. Urey, "MEMS laser scanners: a review", Journal of Microelectromechanical Systems, Vol. 23, pp. 259-275 (2014). 
[2] K. Aksit, O. Eldes, S. Viswanathen, M. Freeman, H. Urey, "Portable 3D laser projector using mixed polarization technique," Journal of Displays, Vol. 8 (10), pp. 582-589 (2012).

[3] K. Aksit, D. Kade, O. Ozcan, H. Urey, "Head-mounted mixed reality projection display for games production and entertainment", Personal and Ubiquitous Computing, Vol. 19, pp. 509-521, (2015).

[4] O. Eldes, K.Aksit, H. Urey, "Multi-view autostereoscopic projection display using rotatory screen," Optics Express, Vol. 21, Iss. 23, pp. 29043-29054 (2013).

[5] M. K. Hedili, M. O. Freeman, and H. Urey, "Microlens array-based high-gain screen design for direct projection head-up displays," Applied Optics Vol. 52, Iss. 6, pp. 1351-1357 (2013).

[6] H. Urey, K. V. Chellappan, E. Erden, P. Surman, "State of the art in stereoscopic and autostereoscopic displays", Proceedings of the IEEE, Vol. 99, pp. 540-555 (2011).

[7] K. Aksit, A. Ghanbari Niaki, E. Ulusoy, H. Urey, "Super Stereoscopy Technique for Comfortable and Realistic 3D Displays," Opt. Lett. (2014).

[8] K. Akşit, S. Olcer, and H. Urey, "56.6 L: Late News Paper: Modular Multi Projection Multi View Autostereoscopic Display using MEMS Laser Projectors," SID Symposium Digest of Technical Papers, Vol.45, Iss. 1, pp. 1067-1069, June 2014

[9] Jannick P. Rolland, Kevin P. Thompson, Hakan Urey, and Mason Thomas, Chapter: 10.4.1"See-Through Head Worn Display (HWD) Architectures," Handbook of Visual Display Technology (2011).

[10] K. V. Chellephan, E. Erden, and H. Urey, "Laser-based displays: a review," Applied Optics, Vol. 49 (Feature issue on Lasers: The first fifty years), Issue 25, pp. F79-F98, 2010.

[11]D. Mengu, E. Ulusoy, and H. Urey. "Holographic Image Projection with Phase Only Spatial Light Modulators via Non-Iterative CGH Computation Method," in Proceedings of Digital Holography and Three-Dimensional Imaging, (2015).

[12]D. Mengu, E. Ulusoy and H. Urey, "Non-iterative phase hologram computation for low speckle holographic image projection", accepted for publication in Optics Express. 